\title{
ON THE INDEPENDENCE OF SOJOURN TIMES IN TANDEM QUEUES
}

\author{
THOMAS M. CHEN, ${ }^{*}$ University of California, Berkeley
}

\begin{abstract}
Reich (1957) proved that the sojourn times in two tandem queues are independent when the first queue is $M / M / 1$ and the second has exponential service times. When service times in the first queue are not exponential, it has been generally expected that the sojourn times are not independent. A proof for the case of deterministic service times in the first queue is offered here.
\end{abstract}

\section{Problem formulation}

Let us consider two tandem FCFS queues, the first queue being $M / D / 1$ and the second queue having exponential service times. Let $\lambda$ denote the rate of Poisson arrivals at the first queue, $T$ the deterministic service time in the first queue, and $\mu^{-1}$ the mean service time in the second queue. The state of the system is defined as the vector of queue lengths at the embedded times $\left\{t_{1}, t_{2}, \cdots\right\}$ when customers move from the first queue to the second queue. Let $n_{1}\left(t_{k}\right)$ be the number of customers left behind in the first queue by customer $k$, and $n_{2}\left(t_{k}-\right)$ be the number of customers found by customer $k$ upon arrival at the second queue. It will be shown that the steady-state probabilities $\pi_{i j}=P\left\{\left(n_{1}, n_{2}\right)=(i, j)\right\}$ do not have a product-form solution, and hence $n_{1}$ and $n_{2}$ are not independent. This will imply that the sojourn times in the two queues, $T_{1}$ and $T_{2}$, are not independent.

\section{Analysis} are

The transition probabilities $P_{i j k l}=P\left\{\left(n_{1}\left(t_{n+1}\right), n_{2}\left(t_{n+1}-\right)\right)=(k, l) \mid\left(n_{1}\left(t_{n}\right), n_{2}\left(t_{n}-\right)\right)=(i, j)\right\}$

$$
P_{i j k l}= \begin{cases}a_{k+1} b_{j+1-l}, & i>0, \quad j \geqq 0, \quad j+1 \geqq l>0, \quad k \geqq i-1 \\ a_{k+1-i} \sum_{m=j+1}^{\infty} b_{m}, & i>0, \quad j \geqq 0, \quad l=0, \quad k \geqq i-1 \\ a_{k} c_{j+1-l}, & i=0, \quad j \geqq 0, \quad j+1 \geqq l>0, \quad k \geqq 0 \\ a_{k} \sum_{m=j+1}^{\infty} c_{m}, & i=0, \quad j \geqq 0, \quad l=0, \quad k \geqq 0 \\ 0, & \text { otherwise, }\end{cases}
$$

where

$$
\begin{aligned}
& a_{k}=\operatorname{Pr}\{k \text { arrivals in } T \text { time units }\}=\frac{(\lambda T)^{k}}{k !} e^{-\lambda T} \\
& b_{k}=\operatorname{Pr}\{k \text { departures in } T \text { time units }\}=\frac{(\mu T)^{k}}{k !} e^{-\mu T} \\
& c_{k}=\operatorname{Pr}\{k \text { departures in } T+t \text { time units }\}=\sum_{m=0}^{k} \frac{\lambda}{\lambda+\mu}\left(\frac{\mu}{\lambda+\mu}\right)^{k-m} b_{m} .
\end{aligned}
$$

Received 4 May 1988; revision received 14 February 1989.

* Postal address: Department of EECS, University of California, Berkeley, CA 94720, USA. 
The steady-state probabilities, assuming that they exist, must satisfy

$$
\pi_{k l}=\sum_{i=0}^{\infty} \sum_{j=0}^{\infty} \pi_{i j} P_{i j k l} .
$$

We assume a product form $\pi_{k l}=\pi_{k}^{1} \pi_{l}^{2}$ and substitute the known steady-state probabilities for the $M / D / 1$ queue (see for example Gross and Harris (1974), pp. 241-243). From the expression for $\pi_{0}^{1} \pi_{l}^{2}$ and the fact $\pi_{l}^{2}=\sum_{k=0}^{\infty} \pi_{k}^{1} \pi_{l}^{2}$, we obtain the set of equations

$$
\sum_{j=l-1}^{\infty} \pi_{j}^{2}\left(c_{j+1-l}-b_{j+1-l}\right)=0, \quad l>0 .
$$

By determining the left-side inverse of the matrix

$$
\boldsymbol{A}_{i j} \begin{cases}c_{j-i}-b_{j-i}, & j \geqq i, \quad i \geqq 1 \\ 0, & 1 \leqq j<i, \quad i \geqq 1\end{cases}
$$

column by column, we can show that $\left\{\pi_{j}^{2}\right\} \equiv 0$ is the only solution of $c_{0} \neq b_{0}$, which is valid under the conditions $\lambda>0, \mu>0$. Hence the steady-state probabilities $\left\{\pi_{i j}\right\}$ cannot have product form.

Lemma 1. For two tandem queues consisting of an $M / G / 1$ queue followed by a $G / M / 1$ queue, $T_{1}$ and $T_{2}$ are dependent if $n_{1}$ and $n_{2}$ are dependent.

Proof. It is shown that $T_{1}$ and $n_{2}$ are dependent when $n_{1}$ and $n_{2}$ are dependent. As noted by Reich (1957), $n_{1}$ and $T_{1}$ are related by

$$
E\left\{z^{n_{1}} \mid n_{2}\right\}=\sum_{n_{1}=0}^{\infty} z^{n_{1}} \int_{0}^{\infty} p\left(n_{1} \mid T_{1}, n_{2}\right) p\left(T_{1} \mid n_{2}\right) d T_{1}=\int_{0}^{\infty} \exp \left(\lambda T_{1}(z-1)\right) p\left(T_{1} \mid n_{2}\right) d T_{1} .
$$

The left-hand side is dependent on $n_{2}$, and so $p\left(T_{1} \mid n_{2}\right)$ depends on $n_{2}$.

Now it is shown that $T_{1}$ and $T_{2}$ are dependent when $T_{1}$ and $n_{2}$ are dependent. Note that $T_{2}$ is the sum of $n_{2}+1$ i.i.d. exponential service times. We find

$$
\begin{aligned}
E\left[\exp \left(-s T_{2}\right) \mid T_{1}\right\} & =\int_{0}^{\infty} \exp \left(-s T_{2}\right) \sum_{n_{2}=0}^{\infty} p\left(T_{2} \mid n_{2}, T_{1}\right) p\left(n_{2} \mid T_{1}\right) d T_{2} \\
& =\sum_{n_{2}=0}^{\infty}\left(1+\mu^{-1} s\right)^{-n_{2}-1} p\left(n_{2} \mid T_{1}\right) .
\end{aligned}
$$

The right-hand side depends on $T_{1}$, so $T_{2}$ and $T_{1}$ are dependent.

\section{References}

Gross, D. and HARris, C. (1974) Fundamentals of Queueing Theory. Wiley, New York.

REICH, E. (1957) Waiting times when queues are in tandem. Ann. Math. Statist. 28, 768-773. 